\title{
Methodological Issues in HRI: A Comparison of Live and Video- Based Methods in Robot to Human Approach Direction Trials
}

\author{
Sarah N Woods, Michael L Walters, Kheng Lee Koay, Kerstin Dautenhahn, Member, IEEE.
}

\begin{abstract}
The main aim of this study was to confirm the findings from previous pilot studies that results obtained from the same Human Robot Interaction (HRI) scenarios in trials using both video-based and live methodologies were comparable. We investigated how a robot should approach human subjects in various scenarios relevant to the robot fetching an object for the subject. These scenarios include a human subject sitting in an open space, sitting at a table, standing in an open space and standing against a wall. The subjects experienced the robot approaching from various directions for each of these contexts in HRI trials that were both live and video-based. There was a high degree of agreement between the results obtained from both the live and video based trials using the same scenarios. The main findings from both types of trial methodology were: Humans strongly did not like a direct frontal approach by a robot, especially while sitting (even at a table) or while standing with their back to a wall. An approach from the front left or front right was preferred. When standing in an open space a frontal approach was more acceptable and although a rear approach was not usually most preferred, it was generally acceptable to subjects if physically more convenient.
\end{abstract}

\section{INTRODUCTION AND BACKGROUND}

$\mathrm{T}$ HIS paper presents research carried out towards the development of a Cognitive Robot Companion for use in a domestic environment as part of the work for the COGNIRON Research project [1]. Our main research is in the area of Human-Robot Interaction (HRI), in particular with regard to socially interactive robots. An excellent overview of socially interactive robots is provided in Fong et al. [2]. We are primarily interested in the human perspective of how robots could be useful in domestic environments; in particular the roles, tasks, and social behaviour that will be necessary for robots to exhibit in order to integrate into normal domestic situations [3].

In order to study human-robot relationships, we have previously run HRI trials using carefully devised test scenarios [4][5][6], where human responses and opinions can be collected using a variety of methods. Other researchers

The work described in this paper was conducted within the EU Integrated Project COGNIRON ("The Cognitive Robot Companion") and was funded by the European Commission Division FP6-IST Future and Emerging Technologies under Contract FP6-002020.

Authors: Sarah N Woods, Michael L Walters, Kheng Lee Koay, Kerstin Dautenhahn. Affiliated to Adaptive Systems Research Group, School of Computer Science, University of Hertfordshire, Hatfield, Herts. UK. Email: \{S.N.Woods, M.L.Walters, K.L.Koay, K.Dautenhahn\}@ herts.ac.uk have also conducted similar HRI trials with human sized robots including Dario et al. [7], Severinson-Eklundh et al. [8], Kanda et al. [9] and Hinds et al. [10]. However, most of these HRI trials to date typically are characterised by relatively small sample sizes and are highly exploratory. HRI studies in general are at a stage where there is not a large body of prior work to guide the design of large scale live trials [11]. Due to the time, resources and personnel required to ensure that valid and reliable results are obtained, before committing to a major trial it is essential to run pilot studies to test the proposed methodology and experimental setting. It would also be advantageous to have a methodology in place where trial predictions could be piloted and tested, before developing and executing full live trials.

To overcome some of these problems in developing live HRI trials, the feasibility of running initial pilot HRI trials using video footage rather than a full live interaction was considered. Although this methodology would certainly be inferior to a live HRI session, it was hoped that it would yield valuable results towards the development of live trials. Kidd [12] found no significant differences between subjects' ratings of personality traits for 'present' and 'remote' (through video) cases of an interaction with a robot head. Shinozawa et al. [13] reported that comparing a robot's recommendation behaviour with an on-screen agent's, for human decision making, depended on the interaction environment and that geometrical consistency between the interaction environment, and robots and on-screen agents was important. Paiva et al. [14] reported that children readily empathised with synthetic (cartoon-like) characters in virtual environments displayed on video screens as they enacted various scenarios. Bailenson et al. [15] found that human subjects reacted to virtual humans in a similar way to real humans in terms of interpersonal distances. Real life videos of robots (which are more realistic than virtual or synthetic characters) are also displayed to subjects through the medium of a video screen so it is reasonable therefore to ask if videos of HRI scenarios can elicit similar responses to real-life human-robot interaction scenarios. Video based HRI trials have the potential advantages to: 1) reach larger numbers of subjects as they are quicker to administer, 2) easily incorporate subjects' ideas and views into later video trials simply by recording extra or replacement scenes into the video based scenarios, 3) carry out trials exposing groups of subjects to an HRI scenario simultaneously, 4) prototype 
proposed live trial scenarios to avoid wasted effort and test initial assumptions, 5) allow greater control for standardised methodologies (i.e. exactly the same robot behaviours, exact trial instructions etc.).

To assess the viability of using video based HRI trials the following main research questions were addressed:

1) Can video based HRI scenarios provide results that are comparable to results obtained from live HRI trials?

2) Under what circumstances would video based trials provide comparable results to live HRI trials?

3) What are the likely limitations of video based trials in gaining valid human responses to HRI scenarios?

We have already gained encouraging results from several small scale pilot studies which have investigated various aspects of how robots should approach and serve human subjects in a socially acceptable way. The specific aspects that the pilot studies considered were: comfortable (socially acceptable) spatial distances for robots approaching standing human subjects [16] and how a robot should approach a seated human [17]. In particular, we have performed pilot studies comparing live HRI trial results with those obtained from video based HRI trials of the same scenario; a robot approaching a seated human subject [17]. Therefore, the main aims of the studies and trials presented here were:

1) To confirm and consolidate the results previously obtained from small scale pilot studies, using more diverse live and video based HRI trials with larger sample sizes.

2) To extend the range of human-robot interaction situations and scenarios studied in these larger scale trials.

\section{EXPERIMENTAL METHOD}

The trial was performed at non-University premises as comments from the participants of the previous pilot study [17] indicated that the use of a converted conference room was not perceived by subjects as homely or neutral, characteristic of a domestic environment. Instead subjects occasionally felt tense and as if their behaviour was being judged, although they were specifically informed that this would not be the case. An apartment near to the University was rented, referred to here as the "Robot House", and the main living room furnished and used as the venue for the large scale trials. Feedback from the participants indicated that they thought the Robot House was not like a laboratory, they felt they were not being tested and the perception of the experimental area was more territorially neutral than a laboratory.

In total, four different scenarios were studied in the trials where a robot approached the subject who was located in the living room:

1) Seated on a chair in the middle of an open space.

2) Standing in the middle of an open space.

3) Seated at a table in the middle of an open space.

4) Standing with their back against a wall.

These particular interactions were chosen as they were typical approach situations which would be encountered in a wide range of fetching and carrying tasks that a domestic robot might be expected to carry out. It is hoped that once the appropriate approach behaviour expected of robots is known, these actions could then be used as 'primitive' robot action components which could be sequenced appropriately into more complex task scenarios involving a robot approaching a human.

The live trials were performed in the living room. The video clips used in the video based HRI trials were recorded in the actual living room used for the live trials, but were shown to subjects in another projection room which was fitted out with an LCD projector and screen. Two of the above HRI scenarios were performed twice for each participant; once each as live trials, and once each as corresponding video trials with clips of the same scenarios shown on a screen in the projection room. Of a total of 42 subjects, the first 20 subjects and the final subject experienced scenarios 1) and 2), the following 21 subjects, scenarios 3) and 4). Therefore, half the subjects each experienced each pair of scenarios.

\section{A. Experimental Setup}

The Robot House was a standard British or Northern European style, two bedroom, ground floor apartment with a small kitchen and a relatively large living room area. The living room area was used for all the live HRI trials, the larger bedroom for the video projection room, and the smaller bedroom used to contain video capture and data logging computers and equipment. The robot used for the trials was a commercially available PeopleBot ${ }^{\mathrm{TM}}$ with standard equipment fitted including a pan and tilt camera unit and a standard short reach lifting gripper, which was adapted to form a simple tray in order to fetch and carry objects as required. The furnishing for the room consisted of pictures on the walls, a three seater sofa, a table, three upright dining chairs and a low coffee table. During the trials, most of the furniture was arranged at one end of the room, to provide a large clear space for carrying out the HRI trials. A chair and/or table were moved to the central position as required for the trial scenarios where the subject was to be seated in the middle of the room or at the table.

The experimental trials were carried out by three researchers: An experiment supervisor, a robot operator and a video and data equipment monitoring operator. The experiment supervisor introduced and explained the trial procedure to each subject. First, a short introduction video was shown to the subject, which provided some background details of our work with robots and HRI. The experiment supervisor then administered consent forms and introductory questionnaires. The first trial was then introduced. Alternate subjects experienced a live HRI trial or a video based HRI trial first. Twenty one subjects experienced two scenarios, 1) and 2) above, in both live and video form and the second set of 21 subjects experienced trial scenarios 3 ) and 4). The trials 
were counterbalanced with regard to Video and Live trial order.

The individual trial scenarios are considered separately in more detail in following sections. It was not possible to allow subjects to be on their own with the robot during the live HRI trials for ethical and safety reasons. Therefore, in order to minimise experimenters in the room, the experiment supervisor handed control of the experiment to the robot operator for the duration of each live trial. The robot operator sat on the sofa at the far end of the room, not in direct line of sight, but still visible to the subject. From post trial interviews of participants of previous pilot studies [17] subjects felt re-assured by the presence of the robot operator. It was explained that the robot operator would only be setting up the robot to a given start position and that the robot would approach them autonomously. All video cameras and data logging equipment were operated and monitored by the monitoring operator from the small bedroom, where the door was closed, out of sight of the subject. Two video cameras recorded each trial; one fixed overhead wide angle camera with an overview of most of the experimental area, and a tripod mounted video camera which recorded a closer view of the subject. The video from the tripod mounted camera was fed directly to a Digital Video (DV) capture computer. The video from the fixed camera was recorded onto DV tape as a precaution in case of DV capture error or failure.

After each HRI approach trial, both video and live, a questionnaire was administered to gain the subjects categorical views of the most preferred and least preferred approach directions, the approach directions judged as most and least efficient and also other information regarding speed of approach and stopping distance. Other questions allowed the subjects to rate efficiency and comfort on five-point Likert scales (using 1 to signify highly negative, 2 fairly negative, 3 as neutral 4 as fairly positive and 5 as highly positive). For example, for rating task efficiency this translated to $1=$ not efficient at all to 5 very efficient, and for comfort this translated to $1=$ very uncomfortable to 5 very comfortable.

During previous pilot trials [17] the robot had a safety stopping distance of $0.5 \mathrm{~m}$. No subjects in those trials felt that this was too close, and other trials investigating human robot approach distances [16] indicate that most humans were comfortable in approaching the PeopleBot robots to within what would be classed as the intimate social space zone (cf. Hall $[18,19]$ ) in the case of human to human approach distances. Therefore, for these trials the robot was set up with a safety stopping distance of $0.2 \mathrm{~m}$ to see if a closer approach by the robot provoked an uncomfortable reaction.

\section{INDIVIDUAL SCENARIO CONDITIONS AND RESULTS}

The four approach trial scenarios are described in more detail here in conjunction with presenting the applicable results and analysis relevant to each scenario. Both the results from the live and video based trials are provided here as well as a comparison between the two sets of results obtained

Forty two subjects were involved in the study, including students and staff members from various disciplines at the University of Hertfordshire. The age of the subjects varied from 18 to 56 years and $36 \%$ were female, $64 \%$ male and $9 \%$ (4 subjects) were left handed or ambidextrous.

All trials were based on the same general situation where the robot was bringing a snack to the human subject. Each time the robot approached from a different relative direction, the subject instigated the approach by speaking to the robot. The actual words were not important, but this was done to allow the subject to be prepared for the robot to actually move towards them. The robot operator used this as a cue to set the appropriate robot approach program into operation. The robot then approached the subject under autonomous control. The operator only took over direct manual control in case of error or emergency stop conditions. This happened only once in all 42 trials, where the robot bumped into a corner of the table. In this case the approach was simply set up again and repeated correctly. For each trial, the approach directions were experienced in a random order, and alternate subjects experienced the video based version before the live version, and visa versa. Questionnaires were used to gain the subjects opinions of the trial after each video based or live approach trial scenario. The details for each trial and results are given below.

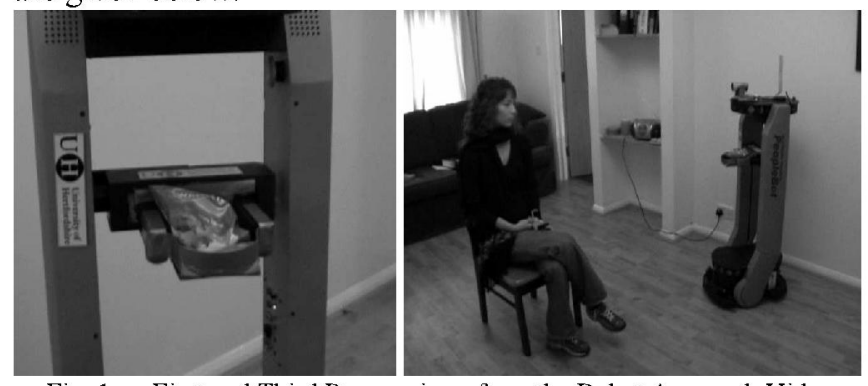

Fig. 1. First and Third Person views from the Robot Approach Video Clips. A typical first person view is shown on the left and a third person view is shown on the right.

For the video based trials, a short clip (in third person perspective) was shown, showing a female actor in one of the relevant scenarios (sitting, sitting at table, standing, standing against a wall) setting the scene by asking the robot to fetch a snack. Then short approach clips that showed the robot approaching from the appropriate relative approach directions (specific to each scenario) were shown to the subject in a random order. Each approach clip had the same format and overall structure; First, a title sequence was shown, which stated what direction the robot would be approaching from. The first part of the clip was a third person view providing an overall view of the robot starting to make an approach from the specified direction, then 
switching to a first person view for the final close approach. See Fig. 1 for examples of the first and third person views.

\section{A. Subject Seated at a Table Scenario: Results and Comparison of Live vs. Video Conditions.}

This Trial scenario involved the robot approaching the human subject who was seated at a table in the middle of the room bringing a snack to the subject. The robot approached the seated human from 5 different directions relative to the subject: from the Front (F), Front-Left (FL), Front-Right (FR), Rear-Left (RL) and Rear-Right (RR). The live trial involved the subject actually sitting on a dining type chair in the living room and asking the robot to approach (see Fig. 2), while the video based trial was shown to the subject who was sitting in front of the projection screen.

\section{1) Results}

Paired t-tests for matched samples were carried out to determine whether there were any significant differences between the subjects approach direction preferences for both live and video based HRIs.

The first set of t-tests refer to the subjects' ratings of the comfort levels experienced for the live vs. video 'seated at the table' scenario. No significant differences were revealed for any of the approach directions (front left, front right, frontal, rear left and rear right). This indicates that subject comfort level ratings for the different approach directions were relatively equivalent between the live and video HRI set-up.

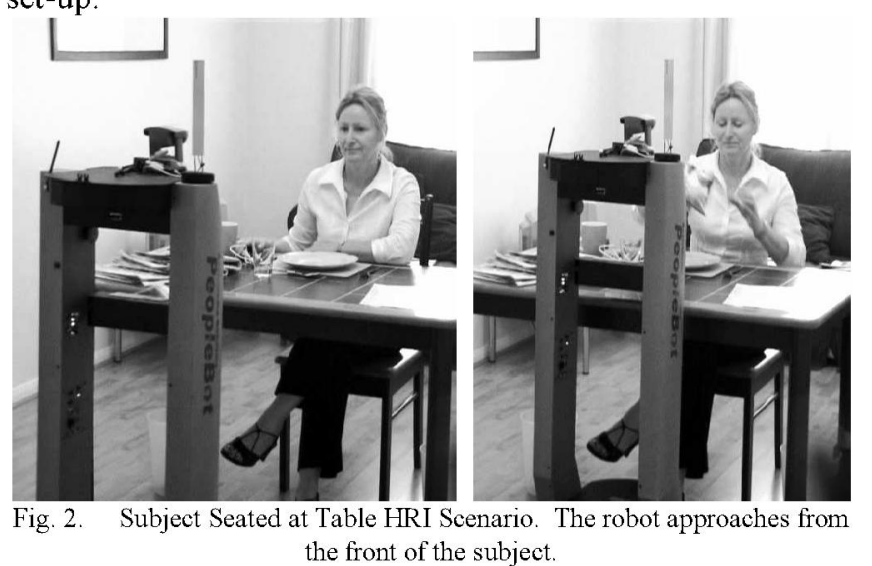

This is further substantiated by the significant Pearson correlations found between the different approach directions for the live and video scenarios (front direct: $\mathrm{p}=.004$; front right: $p<.001$; rear left: $p<.001$; rear right: $p<.001$ ), with the exception of the rear right approach direction $(p=.18)$.

A non-parametric Friedman analysis of variance by ranks was carried out to determine whether there were significant differences between subject comfort rating preferences for the different approach directions. Significant differences were found between the mean rankings for the live approach directions $\left(\mathrm{X}^{2}(4)=26.05, \mathrm{p}<.001\right)$, with subjects rating the front left $(x=4.40)$ and front right $(x=4.35)$ approaches as the most comfortable. Subjects found the rear approaches the least comfortable. Significant differences were also found between the mean rankings of subject comfort levels for the video approach directions $\left(\mathrm{X}^{2}(4)=41.14, \mathrm{p}<.001\right)$. Virtually the same pattern of findings was uncovered with the front right $(x=4.33)$ and front left $(x=3.88)$ approach directions rated as the most comfortable, and the rear approaches were rated as the least comfortable.

Paired sample t-tests for subject responses about the robot's task efficiency for each of the approach directions revealed only one significant difference between the live and video interaction conditions. This was for the rear right approach $(\mathrm{t}(20)=2.35, \mathrm{p}=.03)$, where subjects rated the efficiency higher for the live condition compared to the video condition. Other than this finding, the results were comparable for the live vs. video conditions for all approach directions. Significant correlations between the live and video conditions were revealed between all approach directions with the exception of the front left $(p=.18)$ approach direction (frontal approach $\mathrm{p}=.001$; rear left $<.001$; front right $p=.032$, rear right $p=.001$ )

The Friedman analysis of variance test for subject ratings of robot task efficiency for the live trial was non-significant $\left(\mathrm{X}^{2}(4)=3.23, \mathrm{p}=.520\right)$, indicating that subjects had no preference for the level of task efficiency and the approach direction used by the robot. However, significant differences for robot task efficiency were revealed for the video condition $\left(\mathrm{X}^{2}(4)=16.06, \mathrm{p}=.003\right)$, with all of the frontal approaches being rated as more efficient compared to the rear approaches.

\section{2) Summary of the Seated at Table Condition:}

- Comfort ratings of the different approach directions were comparable across the live and video HRI trials.

- The front left and front right approaches were rated as the most comfortable by subjects, for both the live and video HRI trials.

- The rear approaches were rated as the least comfortable by subjects for both the live and video HRI trials.

- Subject ratings of robot task efficiency were relatively comparable for the live and video HRI trials, with the exception of one approach direction (rear right).

- Subjects did not express an approach direction preference for robot task efficiency for the live trials, but preferred all the frontal approach directions for task efficiency for the video trials.

\section{B. Subject Standing Against a Wall Scenario: Results and Comparison of Live vs. Video Conditions.}

This trial scenario involved the robot approaching the human subject, who was standing with their back against a wall of the room, bringing a snack to the subject. The robot approached the standing human from 3 different directions relative to the subject: from the Front $(F)$, Front-Left (FL) and Front-Right (FR).

The live trial involved the subject actually against the living room wall and asking the robot to approach (see Fig. 3), 
while the video based trial was shown to the subject who was sitting in front of the projection screen.

\section{1) Results}

Paired samples t-tests were carried out to determine whether there were any significant differences between subject comfort ratings for the different approach directions (front, front left, front right), for the standing against a wall scenario, and the live versus video HRI interactions. No significant differences emerged indicating that subject ratings of comfort levels were equivalent across the live and video scenario for the different approach directions.
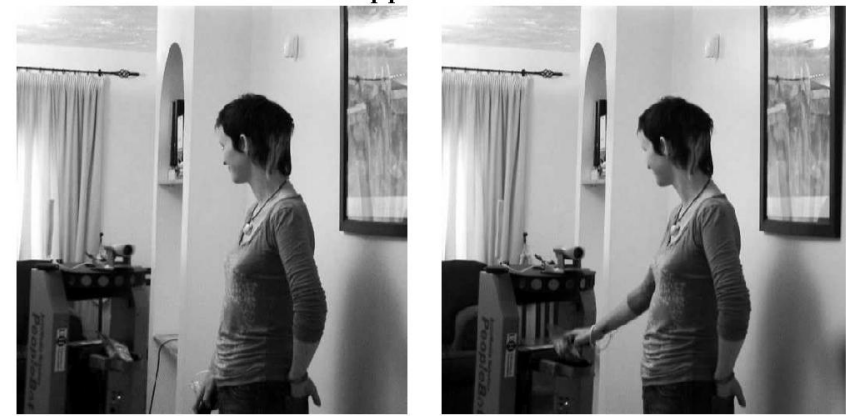

Fig. 3. Subject Standing Against the Wall HRI Scenario. The robot in this trial is approaching from the front right of the subject.

Significant correlations were found for each of the approach directions and the live vs. video set-up, indicating that subject responses were strongly related (left approach, $p$ $=.006$; front direct approach, $p<.001$; right approach, $p<$ $.001)$.

A Friedman analysis of variance by ranks revealed significant differences concerning subject comfort ratings for the different approach directions in the live HRI situation $\left(\mathrm{X}^{2}\right.$ $(2)=9.33, p=.009)$. Subjects rated the front direct approach $(x=3.33)$ as less comfortable compared to the front left ( $x=$ $4.05)$ and front right $(x=4.19)$ approaches. This finding was repeated for the video HRI scenario $\left(X^{2}(2)=9.35, p<\right.$ .009) (front direct approach $x=3.62$; front left $x=4.14$; front right $x=4.29$ ).

Results of paired samples t-tests revealed no significant differences for subject ratings of robot task efficiency for the different approach directions, for the standing against the wall scenario. This suggests that subjects viewed the video and live interaction scenarios equivalently when it came to rating robot task efficiency. Significant correlations were revealed between the live and video set-ups for the left approach direction $(p=.003)$ and the right approach direction $(\mathrm{p}=.001)$. The correlation for the direct frontal approach did not quite meet significance $(p=.14)$.

No significant differences were revealed between subject robot task efficiency ratings for the live robot approaches $\left(\mathrm{X}^{2}\right.$ $(2)=.689, p<.709)$ or the video robot approaches $\left(X^{2}(2)=\right.$ $.542, \mathrm{p}<.763)$. This demonstrates that subjects did not distinguish any overall preferences for a particular approach direction for robot task efficiency, for both the live and video scenarios. Task efficiency ratings ranged from $3.48-4.00$ indicating moderate to high overall task efficiency.

\section{2) Summary of Subject Standing Against the Wall} Conditions:

- Subject comfort level ratings for the different approach directions for the standing against the wall condition were equivalent for the live and video setups.

- The front direct approach was rated by subjects as being the least comfortable for both the video and live conditions.

- Subject ratings of robot task efficiency were equivalent for both the live and video scenarios.

- Subjects did not distinguish any overall preference for the most efficient robot approach direction for both the live and video conditions.

\section{Subject Seated in Middle of Room (Without a Table) Scenario: Results and Comparison of Live vs. Video Conditions.}

This Trial scenario involved the robot approaching the human subject, who was seated in the middle of the room without a table, bringing a snack to the subject. The robot approached the seated human from 5 different directions relative to the subject: from the Front (F), Front-Left (FL), Front-Right (FR), Rear-Left (RL) and Rear-Right (RR). The live trial involved the subject actually sitting on a dining type chair in the living room and asking the robot to approach (see Fig. 4), while the video based trial was shown to the subject who was sitting in front of the projection screen.

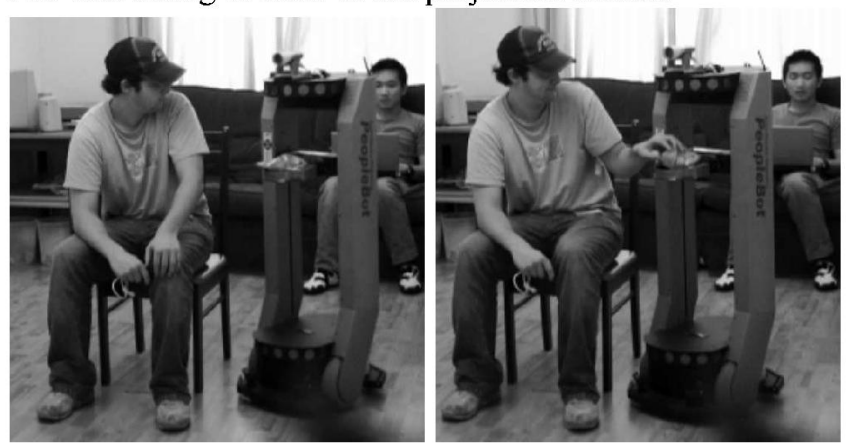
Fig. 4. Subject Seated in Middle of Room HRI Scenario. The robot in this
trial is approaching from the rear left of the subject. 1) Results

Paired samples t-tests revealed no significant differences between subject comfort ratings for the different robot approach directions for the 'seated without a table' condition. This demonstrates that subject responses were relatively equivalent between the live and video scenario regarding comfort ratings. Significant correlations were uncovered between the live and video frontal direct approach $(\mathrm{p}<.001)$, and the rear left approach direction $(\mathrm{p}=.005)$. The remaining correlations did not quite reach statistical significance (front left, $\mathrm{p}=.15$; front right $\mathrm{p}=.10$; rear right, $\mathrm{p}=$.19)

Significant differences were revealed from the Friedman test of variance by mean rankings concerning subject comfort ratings for live approach directions, for the "seated without a 
table' scenario $\left(X^{2}(4)=19.39, p=.001\right)$. Subjects rated the front left $(x=4.24)$ and front right $(x=4.24)$ approach directions as more comfortable than the rear approaches (rear left $x=3.38$; rear right $x=3.62$ ) and the frontal direct ( $x=$ 3.43) approach. Significant differences were uncovered for subject comfort ratings for the video approach directions with a similar pattern as for the live approach directions $\left(\mathrm{X}^{2}\right.$ $(4)=29.41, p<.001)$. Subjects rated the front right $(x=$ 4.30) and front left $(x=4.20)$ approaches as the most comfortable, and the rear (rear left $x=3.15$; rear right $x=$ 3.25 ) approaches as the least comfortable.

With regard to robot task efficiency, no significant differences were found between subject ratings of the live versus video conditions, as a result of the paired samples $t-$ tests. Significant correlations were uncovered between the live and video conditions for all the approach directions (front left, $\mathrm{p}=.003$; frontal approach, $\mathrm{p}<.001$; front right, $\mathrm{p}$ $=.002$; rear left, $p=.001$; rear right, $p=.002$ ). This indicates that subject ratings for the robot task efficiency were significantly related for both the live and video conditions.

Results of the Friedman test were non-significant for both the live and video conditions for subject ratings of task efficiency, indicating that subjects did not display overall preferences for a more or less efficient robot approach direction in both the live and video interactions.

2) Summary of Subject Seated in Middle of Room Condition:

- Subject comfort level ratings for the different robot approach directions were equivalent for the live and video conditions.

- The front left and right approaches were rated by subjects as the most comfortable in both the live and video trials.

- The rear approaches and direct frontal approach was rated as the least comfortable.

- Subjects rated the robot's task efficiency equivalently for the live and video scenarios.

- No significant differences were revealed for the most/least efficient robot approach direction for both the live and video condition.

\section{Standing in the Middle of the Room Scenario: Results and Comparison of Live vs. Video Conditions.}

This Trial scenario involved the robot approaching the human subject, who was standing in the middle of the room, bringing a snack to the subject. The robot approached the seated human from 6 different directions relative to the subject: from the Front (F), Front-Left (FL), Front-Right (FR), Rear (R), Rear-Left (RL) and Rear-Right (RR). The live trial involved the subject standing in the middle of the living room and asking the robot to approach (see Fig. 5), while the video based trial was shown to the subject while sitting in front of the projection screen.

\section{1) Results}

Paired samples t-tests revealed no significant differences between subject comfort ratings for the different robot approach directions, for the live and video conditions. Significant correlations were revealed between the live and video conditions for each of the approach directions (front left, $\mathrm{p}=.014$; front direct, $\mathrm{p}=.002$; front right, $\mathrm{p}=.001$; rear left, $p<.001$; rear right, $p<.001$; rear central, $p<.001$ ). This indicates that subjects rated their comfort levels similarly for each of the different robot approaches for the live and video conditions.

The non-parametric Friedman analysis of variance of mean ranks uncovered significant differences between subject comfort ratings for the different approach directions, for the live condition $\left(\mathrm{X}^{2}(5)=72.36, \mathrm{p}<.001\right)$. Subjects clearly felt the least comfortable with the rear central approach direction $(x=1.86)$, and were the most comfortable with the front left $(x=4.24)$ and front right $(\mathrm{x}=4.38)$ approaches. The same pattern of findings emerged for the video condition $\left(\mathrm{X}^{2}(5)=\right.$ $48.79, \mathrm{p}<.001)$, with subjects rating the rear central approach as the least comfortable $(\mathrm{x}=2.14)$, and the front left $(\mathrm{x}=4.14)$ and front right $(\mathrm{x}=4.29)$ approaches as the most comfortable.
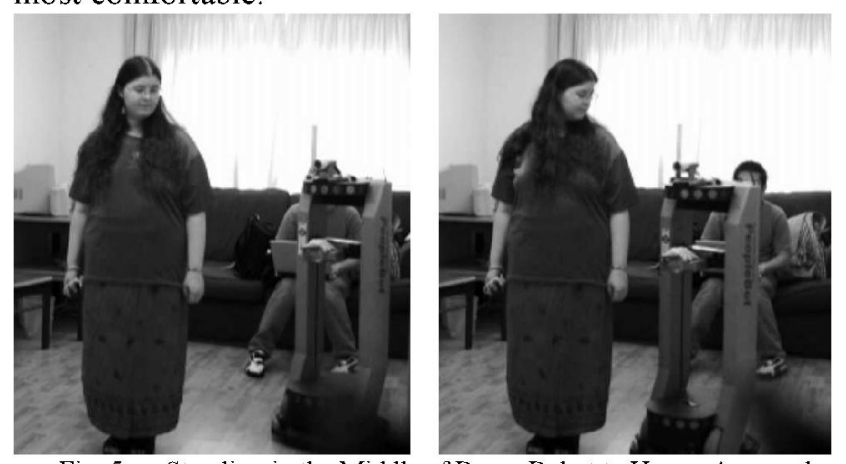

Fig. 5. Standing in the Middle of Room Robot to Hunan Approach Scenario. The robot is approaching in this live HRI trial from the rear left of the subject.

No significant differences emerged from the matched $t$ tests, for subject ratings of the robot task efficiency for each of the robot approach directions, between the live and video conditions. Significant correlations were found between the live and video conditions, for each of the robot approach directions and task efficiency (front left, $\mathrm{p}=.05$; front direct, $\mathrm{p}=.019$; front right, $\mathrm{p}=.028$; rear left, $\mathrm{p}=.041$; rear right, $\mathrm{p}$ $=.001$; rear central, $\mathrm{p}=.001$.

The Friedman test was significant for the live condition, indicating that subject ratings of robot task efficiency varied for the different approach directions $\left(\mathrm{X}^{2}(5)=32.46, \mathrm{p}<\right.$ $.001)$. The rear central $(x=2.57)$ approach was rated by subjects as being the least efficient, and the frontal approach $(x=4.10)$, front right $(x=3.95)$, and front left $(x=3.76)$ approaches were rated as the most efficient. Significant differences in the mean rankings were also revealed for the video condition $\left(\mathrm{X}^{2}(5)=37.42, \mathrm{p}<.001\right)$, with subjects rating the rear central $(x=2.67)$ approach as the least 
efficient, and the frontal approach $(x=4.19)$, front left ( $x=$ $3.86)$ and right $(x=3.86)$ approaches as the most efficient.

2) Summary of the Standing in the Middle of a Room Condition:

- Subject comfort ratings for the different robot approach directions were equivalent for the live and video conditions.

- Subjects rated the rear central approach as the least comfortable, and the front left and right approaches as the most comfortable, for both the live and video conditions.

- Subjects rated the robot task efficiency for the different approach directions similarly for the live and video conditions.

- The rear central approach was rated as the least efficient, and the frontal, and front left and right approaches were rated as the most efficient.

\section{OVERALL SUMMARY OF FINDINGS}

To summarise, consistent findings were revealed comparing live versus video scenario conditions, based on results from our study investigating people's preferences for different robot approach directions in different scenarios and scenarios. The one exception was for robot task efficiency for the 'seated at the table' scenario, where subjects expressed an approach direction preference for task efficiency for the video condition, but not for the live condition. Overall, the front left and right approaches were rated by subjects as the most comfortable for all the different scenario scenarios. The rear approaches and front direct approaches were rated as being the least comfortable across different scenario scenarios.

With regards to robot task efficiency, subjects frequently did not distinguish a particular approach direction preference. However, there were a few exceptions. For the "seated at a table' condition, subjects did not express an approach direction preference for robot task efficiency for the live condition, but rated the frontal approaches as the most efficient from the video trials. The other exception was for the 'standing in the middle of the room' scenario, where subjects did distinguish between robot task efficiency, with the rear central approach being rated as the least efficient and the frontal approaches as the most efficient. In particular, subjects preferred the direct frontal approach for task efficiency. This is in contrast to the other scenario scenarios, but a possible reason could be due to the fact that the subject was standing and would have been taller than the robot, therefore not finding the robot intimidating in any way. This is in contrast to the seated conditions, where the subjects were shorter than the robot, and it would be harder to escape the situation, therefore leading some subjects to rate the direct frontal approach as aggressive and invasive into their personal space. A distinction was also evident for the 'standing in the middle of the room' and the 'standing against the wall' scenarios for the frontal approach. In the standing against the wall condition, subjects rated this as somewhat uncomfortable, but this was not the case for the standing in the middle of the room condition. This could again relate to the feeling of safety with the robot as it would be harder for a subject to escape the standing against the wall condition compared to the standing in the middle of the room condition.

\section{A. Robot Behaviour Design Implications}

Based on our findings, the following design implications for robot behaviour are suggested: A distinction should be made between standing and sitting scenarios. For seated scenarios, the robot should avoid using a direct frontal approach. However, this could be used for situations where the subject is standing but not backed into a wall. Taking account of typical humans' preferences, a direct frontal approach should be avoided when the human is seated, even at a table. A robot should approach a person from the front left or right when delivering an item such as a snack.

With regards to robot physical task efficiency for delivering an item such as a snack, the robot should use the frontal approach directions where physically feasible. The robot should generally avoid using rear approaches, as subjects often found these approaches uncomfortable. However, frequently an approach direction preference was not strongly expressed, indicating that in cases where task efficiency is paramount, rear approaches could be used by the robot.

\section{CONCLUSIONS}

This study indicates some important advances for HRI studies, as it provides further statistical evidence reinforcing results from previous studies where subject ratings for robot to human approach directions were broadly equivalent for both live and video based HRI trials in the majority of cases. This suggests that HRI studies could use videotaped scenarios as opposed to live interactions for new exploratory studies. This would avoid many of the associated costs of having to design and prepare live HRI trials that may not deliver relevant findings. Therefore, videotaped HRI interactions could provide a very suitable method for developing and trying out new innovative studies that are in the pilot phase of testing. Naturally, there are numerous limitations of using video footage for HRI studies, and we are by no means suggesting that they should be a replacement for live HRI studies. It can be expected that the more interaction between a robot and a subject in a trial, the less suitable video trials will be, due to an increased importance of aspects of embodiment, dynamics and contingency of interaction (see Woods et al. [17] for further discussion of these issues). However, for the particular research questions that we consider in the scenario of robot and human social distances and approach directions, contingency of robot and human movements plays a less crucial role and thus lend 
themselves to investigations of video trials. Further studies need to investigate the range of non-interactive to interactive human-robot encounters in more detail with respect to the suitability of video trials.

This study has extended the findings obtained from the single scenario studied in the pilot studies to four fundamental HRI scenarios which may occur in a typical robot serving or object fetching task scenario with standing and seated humans. It has indicated some general social and physical rules that should be incorporated into robot approach behaviour when interacting with humans. Different social approach rules apply depending whether the interacting human is sitting, standing in the open or against a wall or obstacle. The analyses has identified weaker social approach rules giving behaviour which may be overridden in case of physical task convenience and also some social approach behaviour which should be strongly followed even at the expense of physical task efficiency.

Much data obtained from this study awaits further analysis and work will continue to gain further insights from the data records. It would also be desirable to extend the range of HRI scenarios studied in order to provide more generally applicable social behaviour rules, devise more 'primitive' robot action components and the appropriate contexts where the rules apply.

\section{ACKNOWLEDGMENT}

Many thanks go to our colleagues for participating in our trials; in particular to Mike Blow for his narration skills, and help in producing the HRI video scenarios.

\section{REFERENCES}

[1]. COGNIRON website. www cogniron org. Last accessed June 2006.

[2]. T. Fong, I. Nourbakhsh, and K. Dautenhahn, "A survey of socially interactive robots". Robotics and Autonomous Systems 2003, 42. 143166.

[3]. K. Dautenhahn, S. Woods, C. Kaouri, M. Walters, K. L. Koay, I. Werry, "What is a robot companion - Friend, assistant or butler?", Proc. IEEE IROS 2005, (Edmonton, Canada 2005), 1488-1493.

[4]. R. te Boekhorst, M. L. Walters, K. L. Koay, K. Dautenhahn, C. L. Nehaniv, "A study of a single robot interacting with groups of children in a rotation game scenario". Proc. IEEE CIRA 2005, (Finland, 2005), $35-40$.

[5]. M. L. Walters, K. Dautenhahn, R. te Boekhorst, K. L. Koay, C. Kaouri, S. N. Woods, C. L. Nehaniv, C., Lee, D. and Werry, I., "The influence of subjects' personality traits on personal spatial zones in a human-robot interaction experiment". Proc. IEEE RO-MAN 2005, (Nashville, USA, 2005), 347-352

[6]. K. Dautenhahn, M. L. Walters, S. N. Woods, K. L. Koay, C. L. Nehaniv, E. A. Sisbot, R. Alami, T. Simeon. „How May I Serve You? A Robot Companion Approaching a Seated Person in a Helping Context". Proc. ACM HRI 2006. (Salt Lake City, USA, 2005), 172179

[7]. P. Dario, E. Guglieimelli, C. Laschi, "Humanoids and personal robots: Design and experiments". Joumal of Robotic Systems 2001, 18 (12). 673-690.

[8]. K. Severinson-Eklundh, A. Green, H. Hüttenrauch, "Social and collaborative aspects of interaction with a service robot". Robotics and Autonomous Systems 2004, 42. 223-234.
[9]. T. Kanda, T. Hirano, D. Eaton, H. Ishiguro, "Interactive robots as social partners and peer tutors for children: A field trial". Human Computer Interaction 2004,19 (1-2). 61-24.

[10]. P. Hinds, T. Roberts, L. Jones, "Whose job is it anyway? A study of human-robot interaction in a collaborative task". Human Computer Interaction 2004, 19. 151-181.

[11]. M. L. Walters, S. N. Woods, K. L. Koay, K. Dautenhahn, "Practical and methodological challenges in designing and conducting interaction studies with human subjects". Proc. AISB'05 Symposium on Robot Companions Hard Problems and Open Challenges in Human-Robot Interaction, (14-15 ${ }^{\text {th }}$ April 2005, University of Hertfordshire, UK), 110120 .

[12]. C. D. Kidd, "Sociable robots: The role of presence and task in humanrobot interaction". MSc Thesis 2003, Massachusetts Institute of Technology, Massachusetts.

[13]. K. Shinozawa, F. Naya, J. Yamato, K. Kogure, "Differences in effect of robot and screen agent recommendations on human decision-making". IJHCS 2001, 62 (2). 267-279

[14]. A. Paiva, J. Dias, D. Sobral, R. Aylett, S. Woods, L. Hall, C. Zoll, "Caring for Agents that care: Building empathic relations with synthetic agents", in Proc. AAMAS Autonomous Agents and Multi Agent Systems, (Columbia University, New York City, USA, 2004) $194-201$.

[15]. J. N. Bailensen, J. Blascovich, A. C. Beall, J. M. Loomis, "Interpersonal distance in immersiver virtual environments". Personality and Social Psychology Bulletin, 29, 2003, pp. 819-833.

[16]. M. L. Walters, K. Dautenhahn, K. L. Koay, C. Kaouri, R. te Boekhorst, C. L. Nehaniv, I. Werry, D. Lee, "Close encounters: Spatial distances between people and a robot of mechanistic appearance". Proc. IEEERAS Int. Conf. on Humanoid Robots (Humanoids2005), (Tsukuba, Japan, 2005), pp. 450-455

[17]. S. Woods, M. Walters, K. L. Koay, K. Dautenhahn. „Comparing Human Robot Interaction Scenarios Using Live and Video Based Methods: Towards a Novel Methodological Approach". Proc. of the 9th International Workshop on Advanced Motion Control, (AMC'06), (Istanbul, Turkey, March 27-29, 2006), 750-755

[18]. E.T. Hall, The Hidden Dimension: Man's Use of Space in Public and Private. The Bodley Head Ltd, London, UK. 1966

[19]. E. T. Hall, Proxemics. Current Anthropology 9(2-3), 1968. 